\title{
Review of emerging methods and techniques for arterial pressure and flow waves acquisition and analyses
}

\begin{abstract}
Background: A brief review of the principles and tools for arterial pressure measurements is presented. The methods are based on compression of an artery like brachial, radial, digital or some other arteries, that go back to the pulse diagnostics of oriental medicine, decompression Riva-Rocci method and the first sphygmograph invented by E. Marey. Existing tools for the pulse palpation and arterial pressure auscultation are discussed from the point of view of their accuracy and reliability. Applanation tonometry and light absorption methods and their implantation into the lightweight wearable devices are discussed. Advantages and disadvantages of different methods are formulated. It is shown the direct intra-arterial pressure measurements via a catheter remains the only reliable for anaesthetized, postoperative and patients of the cardiac intensive care unit, and persons with cardiovascular diseases, obesity, faint pulse, arrhythmia and some others. Despite increased interest in non -invasive pressure monitoring, many commercially available tools have failed to satisfy the AAMI standards. The systems for continuous noninvasive arterial pressure monitoring based on photosensors are reviewed. Accuracy and reliability of portable units for pulse oxymetry are discussed. The recently developed mobile apps technologies and microchips are promising future technologies for continuous health monitoring based on the multisensory design, though nowadays their accuracy is not satisfactory.
\end{abstract}

Volume 4 Issue 4 - 2018

\author{
Natalya Kizilova \\ Warsaw University of Technology, Poland
}

Correspondence: Natalya Kizilova, Institute of Aeronautics and Applied Mechanics, Warsaw University of Technology, Nowowiejska st., 24, 0-665, Poland, Tel +48 222347444 , Email n.kizilova@gmail.com

Received: April 05, 2018| Published: July 18, 2018

Keywords: arterial pressure, blood flow, pressure/flow measurements, signal analysis, medical diagnostics, biomechanics.

\section{Introduction}

Arterial blood pressure (AP) is the physical parameter that, together with body temperature, is the most frequently measured parameter in hospitals and at home for medical diagnostics purposes. The principles of the AP measurements are based on the physical mechanisms and techniques that originated in ancient Oriental medicine, then in the medieval times, and have been reinvented and improved in XIX -XX centuries. During the last decades the tendency to miniaturization, simplification, and automatization of the BP measurement devices with simultaneous rise in their sensitivity and reliability is observed. ${ }^{1-4}$ Recently more traditional medical devices have been implemented as mobile apps systems for everyday home monitoring of the body health parameters like temperature, ${ }^{5-7}$ glucose concentration, ${ }^{8-11}$ quality of sleep, ${ }^{12-13}$ attack of asthma ${ }^{14-16}$ or epilepsy, ${ }^{17}$ heart sounds ${ }^{18}$ and electrocardiogram, ${ }^{19}$ hemoglobin level ${ }^{20}$ or alcohol uptake ${ }^{21}$ in blood, and AP. ${ }^{22-25}$ Such applications are essential for health state monitoring in chronic patients, to prevent the asthma, epilepsy or heart attack, sleep apnea, hyperglycemic shock and other acute health states. Both standard clinical and personal mobile techniques have been developed based on the models of a standard healthy human being. ${ }^{6,18}$ That is why their use in young or elderly individuals, patients with some specific disease, sportsmen and some other groups is conventional and produce non-repeatable and erroneous data. Therefore, the problem of sensitivity and reliability of the BP equipment and the physical principles used in them must be revisited and updated to satisfy more groups of patients and healthy individuals. In this paper a brief historical review of existing methods and physical principles of AP estimation is presented. Modern techniques for noninvasive BP measurements based on palpation (Riva-Rocci) and auscultation (Korotkoff) methods, and continuous noninvasive arterial pressure (CNAP) measurements photoplethysmography (PHP) and applanation tonometry (AT), their benefits and disadvantages are discussed. The existing Mobile Apps technologies and the results of their testing studies are presented, and the perspectives of their future development are circumscribed.

\section{Historical review of the blood pressure measurements}

Traditional Chinese medicine appeared at $\sim 2500 \mathrm{BC}$ as a system for health diagnostics and treatment based on, among others, arterial pulse parameters palpated at radial, temporal, and some other arteries ${ }^{26}$ Then the method has been transferred with different modifications into the Traditional Tibetan, Persian, Greek and Roman medicine. Famous Persian physician Avicenna (980-1037), brilliant Roman physician Galen (130-200 AD) and their followers were skillful in pulse diagnostics, among other techniques. Ancient doctors estimated pulse strength, rate, and rhythm; they could diagnose the general state of the body and 12 internal organs (Figure 1). The pulse strength corresponded to the AP, while pulse rate was estimated as number of pulses during one breathing cycle of the doctor. In 1595 brilliant Italian scientist Galileo Galilei discovered the mathematical pendulum and the proportion between its length $\mathrm{L}$ and period $\mathrm{T}$ of oscillations. Now we know that $\mathrm{T}=2 \pi \mathrm{L} / \mathrm{g}$, where $\mathrm{g}$ is the gravitational constant. According to the biography composed by his 
student V. Viviani, Galileo used the pendulum with a permanent length for the arterial pulse measurements. He changed the length until the period of oscillations of the pendulum coincided to the frequency of the examined pulse. In that way, Galileo proposed the first quantitative measure of the pulse waves. ${ }^{27}$ First direct measurement of AP has been carried out by English clergyman Stephen Hales in 1730's. He inserted a vertical long fine tube into the incised artery of a horse and measured the height to which the column of blood rose. In that way, the first measurements have been done in $\mathrm{cm}$ or, as we could say now, in $\mathrm{mm}$ water column. Hales conducted the pressure measurements in different domestic and wild animals, calculated the stroke volume and blood flow velocity in larger arteries and capillaries. His measurement data have been published in 1733 in his «Statistical essays». ${ }^{28}$ The second volume described his experiments on animal physiology including the measurement of the "force of the blood", i.e. blood pressure. He discovered the blood rose up to 8 feet 3 inches in the femoral artery of a mare, while it was 12 inches only in the femoral vein. Based on his statistical data, Hales predicted the AP in humans as $\sim 7.5$ feet (or $\sim 175 \mathrm{~mm} \mathrm{Hg}$ ).

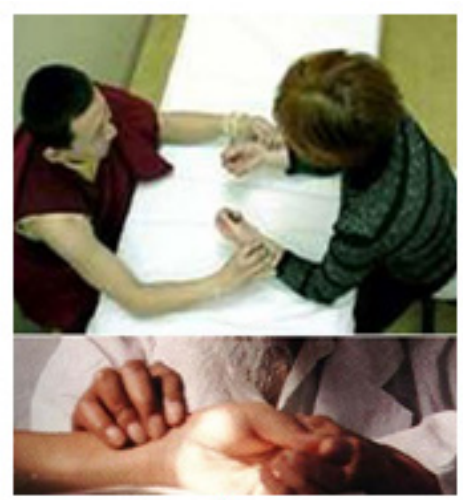

A

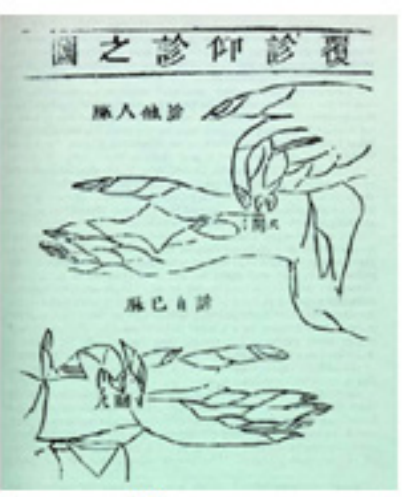

B
Figure I Pulse palpation on the radial arteries at the wrist $(A)$ and six locations for superficial and deep compression of the arteries (B).

The relationships between the AP and blood flow through the vessels have been studied some a hundred years later by famous French physicist Jean Marie Poiseuille. In 1828 during the work over his doctoral thesis "The force of the aortic heart", Poiseuille invented the U-tube mercury manometer ${ }^{29}$ and used it to decrease the volume of blood needed to fill in the measuring tube in comparison to the S. Hales' method. Therefore, Poiseuille measured pressures in the arteries of horses and dogs in $\mathrm{mm} \mathrm{Hg}$. The modified version of his manometer, named the Poiseuille-Ludwig hemodynamometer, was used in hospitals and medical institutions until the end of 1960s and to this day the AP being measured in $\mathrm{mm} \mathrm{Hg}$ by the mercury manometer, remains the golden standard for testing the new AP techniques and designs. J. Poiseuille used his hemodynamometer in the detailed experiments on the fluid flows through fine tubes including the blood flow through metal, glass and other rigid tubes, and segments of blood vessels. In 1840 he formulated his experimental dependence Q $\sim$ PR 4 / $\mathrm{L}$, where $\mathrm{P}$ is the pressure difference at the ends of the tube, $\mathrm{R}$ and $\mathrm{L}$ are the tube's radius and length, $\mathrm{Q}=\mathrm{VS}$ is the volumetric flow rate that is the product of the mean flow velocity $\mathrm{V}$ and the lumen area $\mathrm{S}$. The very first direct measurements of the AP in humans have been carried out in 1856 by French physician A. Favre. He introduced the open end of the hemodynamometer into the arteries of extremities (brachial in the arm and femoral in the leg) just before their amputation and found the mean AP (MAP) was $\sim 120 \mathrm{~mm} \mathrm{Hg}^{30}$ that was significantly lower and more realistic than the value predicted before by S. Hales. Lately the mercury-filled tube has been also used for indirect AP measurements.

The first non-invasive device called sphygmograph for the AP measurements has been designed in 1854 by German physiologist Karl von Vierordt. ${ }^{31}$ It was based on the counterforce measurement and consisted of weights and levers for the artery compression and the weight estimation. In $1876 \mathrm{~S}$. Von Bash proposed a compression method for quantitative AP estimations. He compressed the radial artery of human by an inextensible bag connected to the mercury manometer. He filled in the bag by water until the pulse in the radial artery disappeared. At this moment, the hydrostatic pressure in the bag was assumed to represent systolic AP (SAP). ${ }^{32}$ In 1859 French physiologist Etienne Jules Marey in collaboration with the physiologist Auguste Chauveau and the watch manufacturer Breguet, developed a wearable design for sphygmograph (Figure 2A) to measure the pulse. Marey pioneered in long-time continuous measurements and recordings of the physiological signals by using the rotating cylinder (drum) with a stripe of paper wrapped around it. The ink pen attached via the lever to the counterforce system, left continuous pressure curves on the paper continuously unwrapped by the rotating drum. In 1876 Marey designed a measuring tool, consisted of a rubber sleeve in a rigid cylinder, connected to a mercury manometer by one end and to a pump by another end. When a finger or wrist was inserted into the water-filled sleeve and sealed there by water pumping, weak pressure oscillations in the sleeve have been detected. ${ }^{32}$ In 1902 a French cardiologist Pierre Potain improved this instrument by replacing the mercury manometer by a gauge and substituting water by air. Similar design is used in the modern AP measuring systems (Figure 2B). The next essential improvement of the method was invention by Scipione Riva-Rocci in Italy (1896) and Hill and Barnard in England (1897) a cuff, which was attached around the upper arm and, being pressurized, occluded the brachial artery (Figure 3A). Riva-Rocci developed the decompression method for the diastolic arterial pressure (DAP) measurements. $31 \mathrm{He}$ compressed the brachial artery until complete suppression of the blood flow in it and then slowly decompressed the artery until the first pressure oscillations become palpated downstream of the compression place. As it was shown in 1901 by German pathologist Von Recklinghausen, the cuffs used by Riva-Rocci were too narrow that introduced noticeable errors in the AP values. ${ }^{32}$ In 1899, German physician G. Gärtner introduced a method to determine systolic blood pressure in a finger. First, the finger got anemic by an elastic bandage; then the narrow cuff was applied and inflated. Then the bandage was removed and the cuff was slowly deflated. The cuff pressure at which the finger became flushed, was identified as SAP. ${ }^{33}$ Gärtner's method has been known as the blush or flush method..$^{32} \mathrm{He}$ also developed a method of venous blood pressure estimation by the vertical location of hands at which the veins at the outer surface of palm is observed to collapse, while the palm was slowly risen from the level of heart. Then the distance between the levels of palm and that of the atrium measured in millimeters, gave the venous pressure; thus the vein itself was used as a manometer

The modern approach to the non-invasive quantification of both CAP and DAP has been developed by Russian doctor N. Korotkoff who discovered (in 1905) appearance of sounds (murmurs) in the collapsed empty artery which he listened by a child stethoscope. ${ }^{31-33}$ 
The stethoscope was invented in 1819 by French doctor R. Laennec (Laennic's stethoscope) and since then it was widely used by physicians for listening of the respiratory murmurs, heart beats and other sounds. Korotkoff carried out a series of AP measurements on animals using a Riva-Rocci sleeve, mercury manometer and child stethoscope for his MD thesis. According to the Riva-Rocci method, he raised cuff pressure until it stopped the blood flow in the arm; the SAP was indicated by palpating the radial artery pulse. Then he slowly deflated the cuff and listened the radial artery pulse by the stethoscope placed on the arm beyond the cuff. He attributed the first audible signals to the DAP value. Korotkoff reported that the auscultatory method gave the DAP values by $10-12 \mathrm{mmHg}$ before the pulse could be palpated on the radial artery. ${ }^{33}$ Therefore, according to the original Korotkoff method, the cuff pressure was taken to indicate SAP, while DAP was achieved when the murmur sounds listened by the stethoscope disappeared. ${ }^{33}$ The Korotkoff auscultatory method was later validated by the intravascular measurements of AP in dogs..$^{34,35}$ The mechanism of the Korotkoff sounds is based on the fast accelerated flow of the blood jet that passes through the collapsed brachial artery while the cuff is deflating, and produced high amplitude oscillations of the arterial wall and mechanical (acoustic) waves propagating through the tissues and amplified by the stethoscope ${ }^{36}$ Contemporary sphygmomanometers consist of a rubber bladder woven inside a fabric and supplied by a pneumatic pump and pressure sensors. The width of the cuff must be at least $40 \%$ of the arm circumference. ${ }^{37}$

In parallel to the auscultatory method, the oscillometric method of AP measurement has been developed starting from the pioneering work by E.J. Marrey. In this method, pressure oscillations were recorded by a pen on a Marey's rotating drum during the gradual cuff inflation and deflation. In 1904 Erlanger modified the method by using the Riva-Rocci cuff around the upper arm instead of fingers (Figure 3B). When the cuff is inflated above SAP, there are no pressure oscillations in the cuff. When during the slow cuff deflation the very first low amplitude oscillations are registered, the SAP is indicated. The maximal amplitude oscillations believed to be the DAP ${ }^{38}$ (Figure 3B)). In almost seventy years later it was shown, this point correspond to the MAP, not DAP. ${ }^{39}$ In 1964 S.B. London ${ }^{40}$ patented a unit for automatic cuff inflation/deflation and acoustic signal detection and analysis. During 1964-1967 the unit was modified and improved; its validation has been carried out on 400 persons. The measurement data have been compared to mercury sphigmomanometer and direct intra-arterial measurements. ${ }^{41}$ Further development of the automated AP detection has been based on the cuffs for finger and wrist based AP recognition.

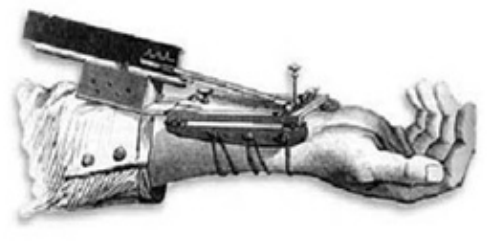

A

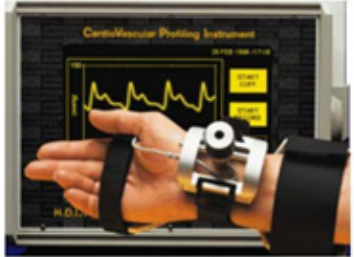

B
Figure 2 Marey's sphygmograph (A) and similar modern instrument Cardiovascular Profiling instrument by hypertension Diagnostics Inc. (B).

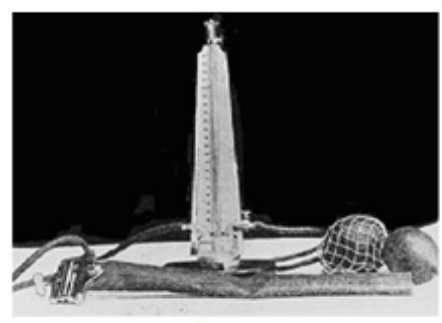

A

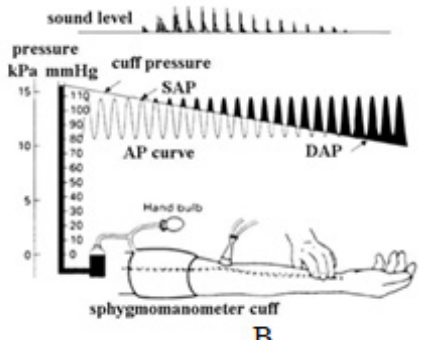

B
Figure 3 Sphygmomanometer by Riva-Rocci with narrow cuff (a) and explanation of the oscillometric method by (B).

\section{Blood pressure measurements and continuous monitoring in modern clinics}

Based on the history of AP measurements and the techniques developed and validated, the following noninvasive methods are nowadays worldwide used for the patient care in hospitals and individually at home:

I. Palpation (Riva-Rocci) and auscultation (Korotkoff).

II. Applanation tonometry.

III. Continuous noninvasive arterial pressure (CNAP) measurements by photoplethysmography.

The golden standards for the new developed devises and approaches are mercury sphygmomanometer and direct (invasive) intra-vessel pressure measurements by pressure sensors installed into a catheter wire.

\section{Palpation and auscultation techniques}

The standard Korotkoff auscultatory method is simple and cheap for everyday blood pressure measurements, but

Widespread banning the use of mercury sphygmomanometers has led to diminishing the role of the method. ${ }^{42}$ Besides, it has the following disadvantages: $:^{30,32,33,43}$

a. It cannot be used in noisy environment.

b. Frequently it is tool-dependent; it is advised to control AP individually by the same tool.

c. The observations differ from invasive measurements in \pm 10 $15 \mathrm{~mm} \mathrm{Hg}$.

d. The technique does not give accurate results for infants and hypotensive patients.

e. In some special cases the diastolic pressure cannot be measured (infinite diastolic tone phenomena).

f. Dependence on the cuff width.

g. Dependence on the body position.

h. Dependence on velocity of calf inflation protocol.

i. Cannot be used for continuous beat-to-beat monitoring. 
Therefore, the difference in the SAP and DAP measured by different sphygmomanometers can reach $5-10 \mathrm{~mm} \mathrm{Hg}$ even in healthy subjects, while in the patients with hypotension, depression, after anesthesia or uptake of some medicines, the difference might be even higher. ${ }^{42,43}$ The Korotkoff murmurs are not audible in noisy environment or in the home use of elderly patients with hearing problems. The auscultatory signals are faint in patients with muscular atrophy and similar impairments. The approach is not applicable for monitoring the training of athletes because after an intense physical load the infinite diastolic murmur can be audible until the very low pressures $\sim 20-30 \mathrm{~mm} \mathrm{Hg}$ which is connected with atypical wave propagation in arteries. The rate at which AP can be recorded is restricted by the inflation and deflation rates of the cuff. Consequently, true beat-to-beat continuous blood pressure monitoring is unlikely by means of this method. ${ }^{42}$ Daily monitoring of AP with a cuff is possible with automatic repetition of the inflation-deflation procedure each $15-20 \mathrm{~min}$, which is more convenient with wrist cuffs (see below). The Riva -Rocci method is helpful is estimation of the AP in noisy environment as far it is based on palpation technique, but only SAP can be measured and the technique does not give accurate results for infants and hypotensive patients. The influence of cuff width on the SAP and DAP has been known since the Riva-Rocci measurements and the minimal cuff width recommended was $\sim 40 \%$ of the perimeter of arm. ${ }^{37}$ It means, the persons with well-developed muscles, fat and obesity need specific wider cuffs for correct measurements, while for the home use the standard ones $(13 \times 35 \mathrm{~cm})$ are commonly used. A narrow cuff can overestimate the $\mathrm{AP}^{44}$ and the British Hypertension Society (BHS) recommends $33 \mathrm{~cm}$ as a critical arm perimeter, ${ }^{45}$ while the American Heart Association (AHA) suggests more detailed widths $\mathrm{W}=13,16$ and $20 \mathrm{~cm}$ for the arm perimeters $27-34,35-44,45-52 \mathrm{~cm}$ accordingly. ${ }^{46}$ The auscultatory signals depend on the body location during the measurements. There is no consensus whether the patient must be sitting or supine during the examination. The latter is essential for the supine patients with spine problems or after a surgery. In the most guidelines sitting is recommend. ${ }^{42,45}$ According to many comparative studies, the sitting and supine position are not essential for the SAP, while a systematic age-related discrepancies have been detected in the DSP values; in sitting position the DAP was $\sim 10 \mathrm{mmHg}$ higher than in the supine one.$^{47}$ It can be easily explained by the higher hydrostatic pressure in the sitting position due to the higher distance to the horizon. Besides, some disease ${ }^{48}$ and arm location ${ }^{49-51}$ also influences the results of AP measurements. There is a progressive increase in the AP in $\sim 5-6 \mathrm{~mm} \mathrm{Hg}$ as the arm is moved down from the horizontal to vertical position, which is also determined by the increased distance between the heart and arm and, thus, the hydrostatic pressure needed for the blood rise. Although in most patients the dynamics of inflation of the sphygmomanometer cuff does not itself change the AP, as it was shown by direct ${ }^{52}$ and indirect (Finapress) ${ }^{53}$ measurements, the sudden inflation may produce an increase in AP up to $40 \mathrm{mmHg}$ in occasional patients. ${ }^{54}$ A rapid stop, repeated measurements may also evoke the vascular respond to the outer compression that produces a short-time increase in the AP. The wrist cuff based wearable equipment become more popular during the last years due to improvements in the sensitivity of the sensors and novel mathematical approaches for the signal recognition, filtering and analysis. Some types of the wrist tools are presented in Figure 4. The cuff is supplied by pressure sensors located distally along the radial artery. When the cuff is pressurized over the SAP value, there are no oscillations of the air pressure in the cuff. During the slow automatic deflation the appearance of the oscillations and their changes in time are measure and analyzed by the software. Usually, the SAP, DAP and heart rhythm are displayed or/ and memorized by the tool. ${ }^{43}$

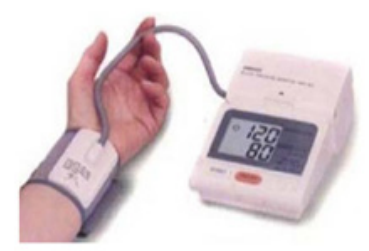

A
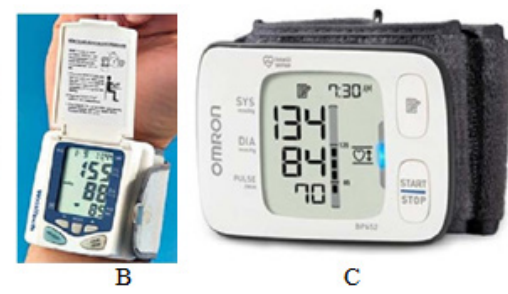

C
Figure 4 Wrist cuff based sphygmomanometers: Omron RI (HEM-60I) (A), WrisTech (B), Omron BP652 7 (C).

\section{Applanation tonometry}

Applanation tonometry (AT) is a method of the AP estimation by continuous measurements of changes in fluid volume due to the pulse wave propagation along a superficial artery like the radial artery (Figure 5). The arterial tonometer is presented by a small plastic chamber $(\mathrm{WxLxH}=2.0 \times 2.0 \times 1.5 \mathrm{~cm})^{55}$ or a hemisphere composed of acrylic materials (plexiglass), and supplied by a flexible membrane. The membrane directly contacts with the artery pressing it to a rigid surface (radial bone). The chamber is filled with a conducting fluid that can move in/out from the chamber depending on whether the artery is collapsed/inflated by the propagating pressure wave. The fluid volume is determined by electric resistivity of the fluid volume that is measured by electrodes. Before use, the tool must be calibrated on the direct or other indirect AP measurements. ${ }^{43,55}$ AT is very sensitive to the muscle contraction and movements of the arm and fingers. For reliability, the arm must be relaxed and the fingers can be fixed by special holders or by the hand of a doctor (Figure 5A). Accuracy of the AT in comparison to the direct intra-arterial measurements on another radial artery was found to be (Bias \pm standard deviation) $-2 \pm 6 \mathrm{mmHg}(17 \%)$ for MAP, $-6 \pm 11 \mathrm{mmHg}(20 \%)$ for SAP, and $+4 \pm 7 \mathrm{mmHg} 23 \%$ ) for DAP (95\% limits of agreement) of the same values as the Korotkoff method. ${ }^{56}$ For the groups of patients with specific disorders and therapy the measuring methodology must be improved further, before being recommended for routine use in this group of patients, for instance, in morbidly obese patients undergoing bariatric surgery. ${ }^{57}$ Recent studies on simultaneous measurements of AP by AT (T-Line 400, Tensys Medical ${ }^{\circledR}$, USA) and the direct intra-artery data in patients with cardiac comorbidities revealed the percentage error $56.8 \%$ for MAP, $42.7 \%$ for SAP, and $75.2 \%$ for DAP. ${ }^{58}$ AT is used for central AP estimations based on the transfer functions radial artery-aorta. ${ }^{59,60}$ AT of the radial artery may not be used in patients of the cardiac intensive care unit. ${ }^{61}$ The difference between the AT (T -line 200) and the direct intra-arterial data was found to be of $+2 \pm 6 \mathrm{mmHg}(-10$ to $+14 \mathrm{mmHg} ; 17 \%)$ for MAP, $-6 \pm 11 \mathrm{mmHg}$ ( -28 to $+15 \mathrm{mmHg} ; 20 \%)$ for SAP, and $+4 \pm 7 \mathrm{mmHg}(-9$ to $+17 \mathrm{mmHg} ; 23 \%$ ) for DAP. Similar studies on cardiac patients with severe cardiac comorbidities revealed the correlation coefficients for MAP, SAP and DAP to be $0.23,0.45$ and 0.06 , respectively; the bias $14.96,4.51$ and $19.12 \mathrm{mmHg}$; the percentage errors 56.8, 42.7 and $75.2 \%$ accordingly. ${ }^{6}$ Automated AP measurement tools were found acceptable for MAP, partially acceptable for DAP, and unacceptable SAP estimations in cardiovascular postsurgical intensive care patients. ${ }^{63}$ Statistical analysis of twelve validations (566 patients; five home, three ambulatory and three office devices) revealed the 
automatic AP monitors are accurate in measuring SAP but not DAP in patients with atrial fibrillation. ${ }^{64}$ Based on AT principle, a watch with an automatic wearable AT sensor implemented in it has been recently developed (Figure 6). The AT sensor measures SAP and DAP, compute the heart rate, radial augmentation index, and restore the central aortic systolic pressure. The wearable modified AT could be very helpful in for fast and continuous AP measurement in hypertonic and postoperative patients, as well as for monitoring the AP during the physical training in elderly persons. Nevertheless, comparative study ${ }^{65}$ of the AP measured by BPro ${ }^{\circledR}$ tonometry sensor and direct intra-artery measurements in the postoperative patients revealed the BPro method is inaccurate in determination of the MAP and SAP based on AAMI (Association for the Advancement of Medical Instrumentation) recommendations. Also when defined by percentage error, the error was out of the acceptable limit of $30 \%$. The method is based on compression of the radial artery by the hemispherical AT sensor (Figure 5B) and the source of mistakes, especially in the home use, could be in the incorrect determination of the artery location and direction of its compression towards the radial bone which are individual.
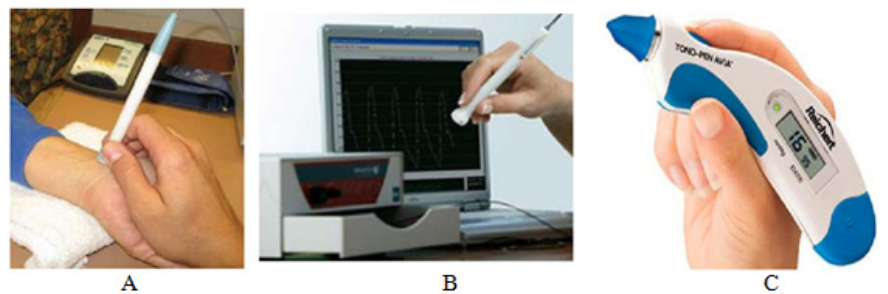

Figure 5 Different types of AT tools with external (A), PC-based (B) and internal screen and software.

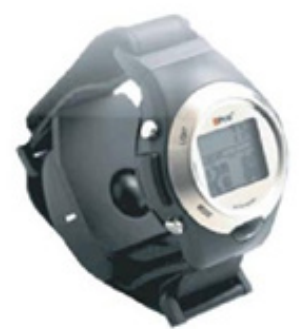

A

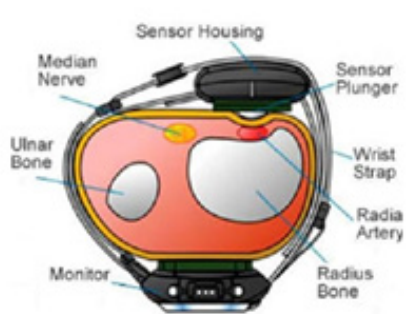

B
Figure 6 Continuous AP monitors BPro $($ (BPRO Ireland Ltd) $(A)$ and its schema (B).

\section{Continuous noninvasive arterial pressure (CNAP) measurements by photoplethysmography}

CNAP is aimed at measuring AP continuously in real-time, without any interruptions like in the Riva-Rocci or Korotkoff methods. Due to high importance and demand for easily applicable and accurate CNAP systems, many research groups, practitioners and manufacturers focus on development and validation of such systems. The most popular CNAP systems are based on pulse oxymetry which illuminates the skin and measures changes in light absorption by the difference in the energy of illuminated and absorbed light. There are also systems based on electroplethysmography. The pulse oxymetry CNAP method was introduced by Czech physiologist Jan Peňáz (1973) with electropneumatic control loop for restoration of AP curves. Pulse oximeters measure finger blood volume $\mathrm{V}(\mathrm{t})$ variations in time. Due to non-lineal elastic and viscoelastic properties of the arterial wall and muscles, the pressure curves $\mathrm{P}(\mathrm{t})$ can not be directly restored from the $\mathrm{V}(\mathrm{t})$ data with sufficient accuracy and reliability. To obtain linear relationship between $\mathrm{V}(\mathrm{t})$ and $\mathrm{P}(\mathrm{t})$, the external counter pressure as high as the pressure inside the artery must be applied. When the external pressure exactly corresponds to the AP, the arterial wall is unloaded and the blood volume in it is constant. Due to that the method is called "vascular unloading technique" or "volume clamp method". In this method a cuff is placed over the finger. Two light emitting diodes produce the beams in the red and infrared (IR) frequencies that propagate through the tissues being partially reflected and absorbed. The transmitted light is measured by a light detector located oppositely to the light source (Figure 7A). In other designs, the reflected light is measured by the detector located at the same side (Figure 7B). The transmission type sensor is currently the mainstream. The lightweight design (Figure 7C) is suitable for long time monitoring. The received light signal is kept constant by the feedback loop controlling the alterable cuff pressure. During systole, when blood volume clasped in the finger increases, the control system increases cuff pressure, too, until the excess blood volume is squeezed out. During diastole, when the blood volume is decreased, cuff pressure is automatically lowered that increases the volume clasped in the finger. Therefore, the intraarterial pressure is equal to the cuff pressure measured by the pressure sensor (manometer).The most popular Finapres (Finger Arterial Pressure) system (Figure 8A) was developed in 1980 by a group from the Netherlands66. The finger arteries are affected by contraction and dilatation in relation to psychological and physical (heat, cold, blood loss, orthostasis) stress that can influence the registered signals. The measurement results are very sensitive to the muscle contractions and finger movements. The latter must be avoided by comforting the patient, covering and holding the hand. In the Finapres technology effects of the smooth muscle contractions are reduced by the builtin PhysioCal algorithm. ${ }^{67}$ Later the Finometer, Portapres (FMS) and Nexfin (Figure 8B) appeared at the market. Recently more complex systems like a family of CNAP monitors have been developed (Figure 8C) ${ }^{68}$ CNAP monitors use the VERIFI-algorithm for correction of the vasomotor tone by means of a fast pulse wave analysis. The algorithm checks typical characteristics of the pulse wave and establishes correct MAP in the finger cuff after every heartbeat. When the vasomotor parameters vary, the needed corrections are computed and introduced immediately, before the next heartbeat. Modern CNAP systems provide information on AP measured by two finger sensors, a wrist sensor and brachial artery cuff (Figure 8C). Information on the instant SAP, DAP and MAP can be obtained (Figure 9A). The long term measurements (Figure 9B) allow determination of the heart rhythm, heart rate variability (HRV), short respiratory and long waves of the daily rhythm (Figure 9C), and pulse waveforms (Figure 9D). Blood pressure variations are helpful in detection of hemodynamic instabilities. Analysis of physiological rhythms is essential in diagnostics of hemodynamic control over AP and blood volume. HRV gives important information on the heart function. The corresponding spectral, fractal, wavelet, Lyapunov exponent, entropy, and other essential diagnostic analyses can be done based on the HR curves. The registered pressure waveforms $\mathrm{P}(\mathrm{t})$ can be used for contour analysis, spectral analysis, computations of the augmentation index, systolic and diastolic areas and other essential parameters needed for 
more detailed pulse diagnostics. ${ }^{69}$ CNAP devices display real-time, continuous arterial pressure waveforms and allow noninvasive beatto-beat arterial pressure measurement.68 Despite increased interest in non-invasive arterial pressure monitoring, ${ }^{69}$ many commercially available technologies have failed to satisfy the limits established for the validation of automatic arterial pressure monitoring by the AAMI for certain groups of patients. According to the ANSI/AAMI/ISO 81060-2:2013 standards, the group-average accuracy and precision are defined as acceptable if bias is not greater than $\pm 5 \mathrm{mmHg}$ and standard deviation is not greater than $\pm 8 \mathrm{mmHg}$. Accuracy of the noninvasive finger cuff CareTaker ${ }^{\circledR}$ has been studied according to the AAMI recommendations. ${ }^{70}$ The correlations of SAP, DAP, and MAP were $0.92,0.86,0.91$, respectively. The Bland-Altman comparison showed a bias of -0.57 (SD 7.34), -2.52 (SD 6.47), 1.01 (SD 5.33) $\mathrm{mmHg}$ accordingly. The polar plot indicates little bias between the two methods $\left(90 \% / 95 \% \mathrm{CI}\right.$ at $31.5^{\circ} / 52^{\circ}$, respectively, overall bias $\left.=1.5^{\circ}\right)$ with only a small percentage of points outside these lines. The 4Q plot indicates good concordance and no bias between the methods. The Finapres technology embodied in several TNO-prototypes and in the Ohmeda 2300 and 2300e Finapres NIBP has been reviewed based on the results of 43 papers published. ${ }^{71}$ It was found the accuracies for SAP, DAP and MAP were ranging from -48 to $30 \mathrm{mmHg}$, from -20 to $18 \mathrm{mmHg}$, and from -13 to $25 \mathrm{mmHg}$ accordingly. Weighted for the number of subjects included pooled accuracies were -0.8 (SD 11.9), $-1.6(8.3)$ and $-1.6(7.6) \mathrm{mmHg}$ respectively. A systematic review and meta-analysis of studies comparing continuous noninvasive arterial pressure monitoring with invasive arterial pressure monitoring has been carried out on 29 studies with 919 patients in total. ${ }^{72}$ The overall random-effect pooled bias and SD were $-1.6 \pm 12.2 \mathrm{mmHg}(95 \%$ limits of agreement -25.5 to $22.2 \mathrm{mmHg}$ ) for systolic arterial pressure, $5.3 \pm$ $8.3 \mathrm{mmHg}$ (-11.0 to $21.6 \mathrm{mmHg}$ ) for diastolic arterial pressure, and 3.2 $\pm 8.4 \mathrm{mmHg}$ (-13.4 to $19.7 \mathrm{mmHg}$ ) for MAP. In 14 studies focusing on currently commercially available devices, bias and SD were -1.8 $\pm 12.4 \mathrm{mmHg}$ (-26.2 to $22.5 \mathrm{mmHg}$ ) for systolic arterial pressure, 6.0 $\pm 8.6 \mathrm{mmHg}$ ( -10.9 to $22.9 \mathrm{mmHg}$ ) for diastolic arterial pressure, and $3.9 \pm 8.7 \mathrm{mmHg}$ (-13.1 to $21.0 \mathrm{mmHg}$ ) for MAP.

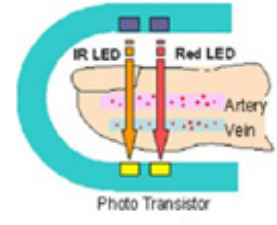

A

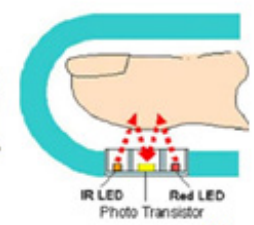

B

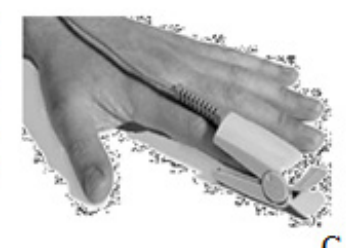

$\mathrm{C}$
Figure 7 Pulse oxymetry sensor of transmission (A) and reflective (B) types, and external view $(\mathrm{C})$.

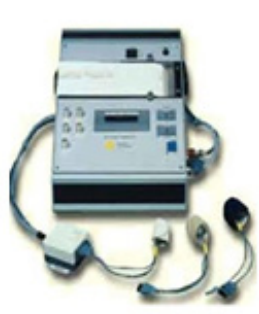

A

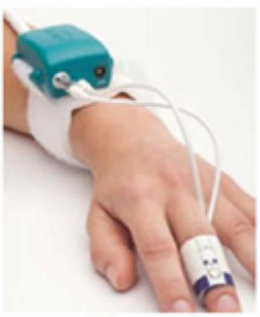

B

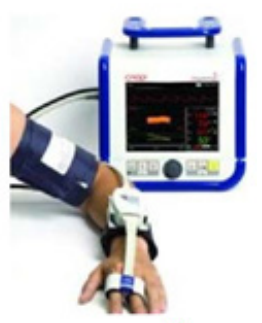

C
Figure 8 Finapress (A), Nexfin (BMEYE B.V., Amsterdam, The Netherlands) (B), and CNAP Monitor 500 (CNSystems, Graz,Austria) (C) systems.
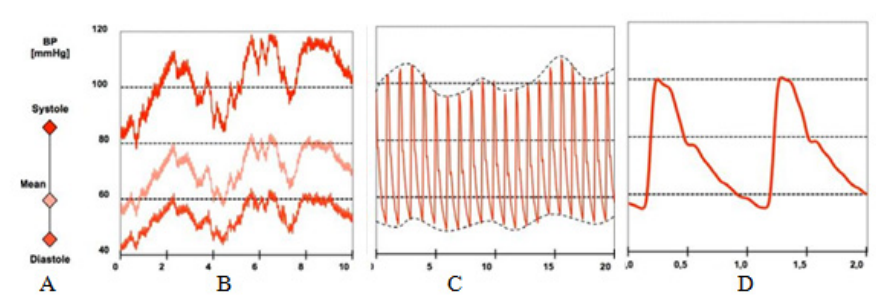

Figure 9 The AP parameters: SAP, DAP, MAP (A), long (B) and short (C) waves, pressure waveforms (D).

\section{Mobile apps for smartphones and telemedicine}

Therefore, obvious progress in the accuracy, reliability and embedded software for analysis of the measured data is observed during the last decade. The attempts to decrease the size of the pulse oxymeters and made them portable and wearable, led to elaboration of the wireless tools (Figure 10). Such tools are portable, allows independent measurements in any suitable time, memorization of the datasets, and distant communication with doctor via sms, mms or e-mail exchange, mobile submission of the measured data to PC via infrared port (IR), and accept ion of new prescriptions from the doctor. During the last years the mobile applications (mobile apps) based on the sensor panels of mobile phones and tablets or interacting with them via IR port become popular. The fingertip-based measurements by special systems equipped with a sensor panel for pressure, temperature and light beam reflection measurements (Figure 11A) (Figure 11B) and mobile phone apps (Figure 11C) can be used for the AP and heart rhythm estimations. The accuracy, reproducibility and reliability of such systems have not been checked yet and, therefore, they are recently attributed as AP pranks. Nevertheless, further improvement of such technology is very promising for AP monitors for home and office use, accumulation of personal datasets for continuous health monitoring and telemedicine. Telemedicine is based on the use of telecommunication and information technologies (IT) for fast and easy urgent and permanent clinical health care from a distance. ${ }^{76}$ Rapid development of IT and telecommunications has created the potential for distantly accessible high -quality expert healthcare to be delivered when and where it is needed. ${ }^{77}$ The first experience of telemedicine is dated by 1950, when several hospitals and university medical centers in Pennsylvania transmitted radiologic images via telephone for diagnostic purposes. ${ }^{76,77}$ Then this technology was developed into a Teleradiology system and used in Canada around Montreal. In 1959 in University of Nebraska the neurological examinations have been transferred to medical students via an interactive television. Mobile health is a newer concept that describes services supported by mobile communication devices, such as wireless patient monitoring devices, smartphones, personal digital assistants, and tablet computers. ${ }^{78}$
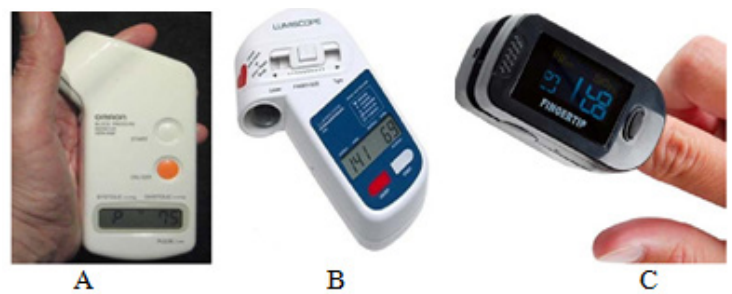

Figure 10 Omron (A), Lumiscope I083N (B), and FingerTip (C) portable AP monitors. 

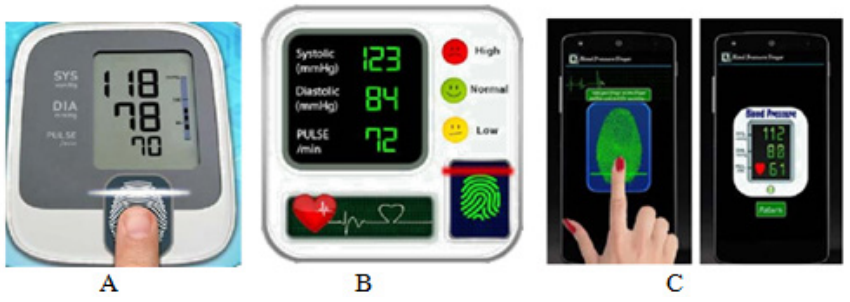

Figure I I Finger Blood Pressure Checker Prank (Superior Technologies, Inc.) (A), Finger BP (SmartApps Ltd.) (B), and Blood Pressure Finger Prank (Google Play) (C) finger AP monitors.

\section{Discussions and perspectives}

In the previous chapters a brief review of the existing techniques of the non-invasive AP measurements in the historical perspective and their implementation in the new generation of tools with easy access and operation, light weight design, low cost, data transition, treatment and analyses has been presented. The novel units are based on the well-known techniques of compression-inflation of the peripheral artery, its wall oscillations or light transmission through it. In spite of noticeable success of the technique, low sensitivity, repeatability and comparison with the 'golden standards' are still reasons for not recommending them by the Association for the Advancement of Medical Instrumentation and the British Hypertension Society. The international protocol requires a total number of 33 subjects in which the validation is performed. For instance, for the validate AP measurement tools at the brachial (Omron ${ }^{\circledR}$ M2, Omron M3, and Omron M6) and wrist (Omron R2) levels the mean differences between the device and mercury readings $2.7 \pm 5.0$ and $-1.4 \pm 3.2$ $\mathrm{m} \mathrm{mHg}$ for systolic and diastolic AP, respectively, using the Omron M2 device, and $1.7 \pm 3.2$ and $-0.9 \pm 2.6 \mathrm{mmHg}$ using the Omron $\mathrm{M} 3,1.6 \pm 2.9$ and $-0.9 \pm 2.5 \mathrm{mmHg}$ using the Omron M6, and $-1.1 \pm 4.8$ and $-0.9 \pm 4.3 \mathrm{mmHg}$ using the Omron R2 have been established. Those values are in perfect correspondence to the 'golden standards' rather than the average AP monitors some 20-30 years ago. ${ }^{69}$ In spite of low accuracy of the existing smartphone-based tools for AP measurements, ${ }^{76-78}$ the high needs of such cheap and accessible technologies will enhance competition in this area and, thus, appearance more sensitive and reliable devices on the market. The measurement principles could be based on not only historically developed ones, but on nanotechnologies for local patient-specific measurements of displacements/light absorption of the radial or other superficial arteries with AP analysis by artificial intelligence.

\section{Conclusion}

Existing technique for the noninvasive AP measurements at home/ office and hospitals are based on registration of pressure oscillations in a pressurized cuff located over a superficial artery (brachial, radial or digit) mostly by pressure sensors or light absorption in the tissues with oscillating perfusion by optical sensors. The first group of tools uses the Riva-Rocci, Korotkoff decompression method and the oscillometric method. During the last decades accuracy and reliability of the tools have been increased significantly. Anyway, despite increased interest in non-invasive AP monitoring, many commercially available technologies have failed to satisfy the AAMI standards especially for the patients with cardiovascular diseases, obesity, faint pulse and some others. Regularly new AP tools are tested for clinical and home use and the ratio of recommended units ranges at $\sim 20-25 \%$ for self measurements and $\sim 60-70 \%$ for ambulatory measurements. ${ }^{73}$ Direct invasive intra -arterial measurements remain the only acceptable for anaesthetized, postoperative individuals and patients of the cardiac intensive care unit.74 This problem demands further development of accuracy of sensors and algorithms for the signal treatment, as well as elaboration of novel principles of AP measurements. Recently an ultrathin, waterproof, cuff less blood pressure sensor has been elaborated. ${ }^{75}$ It is a microchip that can be worn on the wrist, woven into clothes or bed sheets, or integrated into an earpiece. Development of advanced mathematical methods of signal analysis based on artificial neural networks is also promising for future technologies of continuous health monitoring. Such systems must be light and wearable, have multipurpose sensors for AP, temperature, glucose measurements, and communicate with computer and mobile phone for proper signal treatment and analyses, and distant communication with doctors.

\section{Acknowledgements}

None.

\section{Conflict of interest}

Author declares that there is no conflict of interest.

\section{References}

1. Rothfuss MA, Unadkat JV, Gimbel ML, et al. Totally implantable wireless ultrasonic Doppler blood flowmeters: toward accurate miniaturized chronic monitors. Ultrasound in Med \& Biol. 2017;43:561-578.

2. Caldara M, Nodari B, Re V, et al. Miniaturized and low-power blood pressure telemetry system with RFID interface. Proced Engin. 2014;87:344-347.

3. Wu N, Tian Y, Zou X. A miniature fiber optic blood pressure sensor and its application in in vivo blood pressure measurements of a swine model. Sensors \& Actuators Ser B: Chemical. 2013;181:172-178.

4. McMahon C, Mitchell AZ, Klein JL, et al. Evaluation of blood pressure measurement using a miniature blood pressure transmitter with jacketed external telemetry in cynomolgus monkeys. J Pharmacol Toxicol. 2010;62:127-135.

5. De Oliveira Júnior AJ, De Souza SRL, Da Cruz VF. Development of an android APP to calculate thermal comfort indexes on animals and people. Computers Electronics Agricult. 2008;151:175-184.

6. Esposito M, Minutolo A, Megna R, et al. A smart mobile, self-configuring, context-aware architecture for personal health monitoring. Eng Applic Artif Intell. 2018;67:136-156.

7. Werner J. Measurement of temperatures of the human body. Compreh Biomed Phys. 2014;5:107-126.

8. Bandodkar AJ, Imani S, Nuñez-Flores R, et al. Re-usable electrochemical glucose sensors integrated into a smartphone platform. Biosensors \& Bioelectron. 2018;101:181-187.

9. Soni A, Jha SK. Smartphone based non-invasive salivary glucose biosensor. Analyt Chim Acta. 2017; 996:54-63.

10. Ji D, Liu L, Li Sh, et al. Smartphone-based cyclic voltammetry system with graphene modified screen printed electrodes for glucose detection. Biosensors \& Bioelectron. 2017;98:449-456.

11. Li J, Fernando Ch. Smartphone-based personalized blood glucose prediction. ICT Express. 2016;2:150-154.

12. Fietze I. Sleep Applications to Assess Sleep Quality. Sleep Med Clinics. 2016;11:461-468. 
13. Ong AA, Gillespie MB. Overview of smartphone applications for sleep analysis. World J. Otorhinolaryngol. Head \& Neck Surg. 2016;2:45-49.

14. Fedele DA, McConville A, Thomas JG, et al. Applying interactive mobile health to asthma care in teens (AIM2ACT): development and design of a randomized controlled trial. Contemp Clin Trials. 2018;64:230-237.

15. Khasha R, Sepehri MM, Mahdaviani SA, et al. Mobile GIS-based monitoring asthma attacks based on environmental factors. J Cleaner Prod. 2018;179:417-428.

16. Cook KA, Modena BD, Simon RA. Improvement in asthma control using a minimally burdensome and proactive smartphone application. J Allergy Clin Immunol. 2016;4:730-737.

17. Ranganathan LN, Chinnadurai SA, Samivel B, et al. Application of mobile phones in epilepsy care. Int J Epilepsy. 2015;2:28-37.

18. Nguyen HH, Silva JNA. Use of smartphone technology in cardiology. Trends in Cardiovasc Med. 2016; 26:376-386.

19. Guzik P, Malik M. ECG by mobile technologies. J Electrocardiol. 2016;49:894-901.

20. Hasan MK, Haque M, Sakib N, et al. Smartphone-based human hemoglobinl measurement analyzing pixel intensity of a fingertip video on different color spaces. Smart Health. 2018;5-6:26-39.

21. Aymerich J, Márquez A, Terés L. Cost-effective smartphone-based reconfigurable electrochemical instrument for alcohol determination in whole blood samples. Biosensors \& Bioelectron. 2018.

22. İlhan I. Smart blood pressure Holter. Comp Meth Progr Biomed. 2018;156:1-12.

23. Xin Q, Wu J. A novel wearable device for continuous, non-invasion blood pressure measurement. Comput Biol Chem. 2017;69:134-137.

24. Dias Junior A, Murali S, Rincon F, et al. Methods for reliable estimation of pulse transit time and blood pressure variations using smartphone sensors. Microproc Microsyst. 2016;46:84-95.

25. Daponte P, De Vito L, Picariello F, et al. State of the art and future developments of measurement applications on smartphones. Measurement. 2013;46:3291-3307.

26. Ni M. The Yellow Emperor's Classic of Medicine: A New Translation of the Neijing Suwen with Commentary, Boston: Shambhala; 1995.

27. Bedini SA. The pulse of time: Galileo Galilei, the determination of longitude, and the pendulum clock. Fierenze: L.S. Olschki;1991.

28. Hall WD. Stephen Hales: theologian, botanist, physiologist, discoverer of hemodynamics. Clin Cardiol. 1987;10:487-489.

29. Sutera SP. The history of Poiseuille's law. Annu Rev Fluid Mech. 1993;25:1-19.

30. Ghai CL. A textbook on practical physiology. New Delhi-London: Jaypee Brothers Med Publ Ltd; 2013.

31. Roguin A. Scipione Riva-Rocci and the men behind the mercury sphygmomanometer. Int J Clin Pract. 2006;60:73-79.

32. Sorvoja H. Noninvasive blood pressure pulse detection and blood pressure determination. $\mathrm{PhD}$ thesis. University of Oulu. Oulu: Oulu Univ Pess; 2006.

33. Geddes LA. Handbook of blood pressure measurement. NY: Springer Science\&Business Media; 2013.

34. Warfield LM. Studies in auscultatory blood-pressure phenomena. I. The experimental determination of diastolic pressure. Arch Intern Med (Chic). 1912;10:258-267.
35. Macwilliam JA, Melvin GS. Systolic and diastolic blood pressure estimation, with special reference to the auditory method. $\mathrm{Br}$ Med $\mathrm{J}$. 1914;28:693-697.

36. McCutcheon EP, Rushmer RF. Korotkoff sounds. An experimental critique. Circ Res. 1967;20:149-161.

37. Alexander H, Cohen M, Steinfeld L. Criteria in the choice of an occluding cuff for the indirect measurement of the blood pressure. Med Biol Eng Comput. 1977;15:2-10.

38. Howell WH, Brush CE. A critical note upon clinical methods of measuring blood pressure. Boston Med\&Surg J. 1901;145:146-151.

39. Posey JA, Geddes LA, Williams H, et al. The meaning of the point of maximum oscillation in cuff pressure in the indirect measurement of blood pressure. Part 1. Cardiovasc Res Cent Bull. 1969;8:15-25.

40. London SB. Blood pressure monitors. US Patent US3319623A; 1964.

41. London SB, London RE. Critique of indirect diastolic point. Arch Intern Med. 1967;119:39-49.

42. Pickering TG, Hall JE, Appel LJ, et al. Recommendations for blood pressure measurement in humans and experimental animals: Part 1: blood pressure measurement in humans: a statement for professionals from the Subcommittee of Professional and Public Education of the American Heart Association Council on High Blood Pressure Research. Hypertension. 2005;111:697-716.

43. Meyer-Sabellek W, Anlauf M, Gotzen R. Blood Pressure Measurements: New Techniques in Automatic and in 24-hour indirect monitoring. NY: Springer Science \& Business Media; 2012.

44. Ogedegbe G, Pickering T. Principles and techniques of blood pressure measurement. Cardiol Clin. 2010;28:571-586.

45. Petrie JC, O Brien ET, Littler WA, et al. Recommendations on blood pressure measurement. Br Med J (Clin Res Ed). 1986;293:611-615.

46. Perloff D, Grim C, Flack J, et al. Human blood pressure determination by sphygmomanometry. Circulation. 1993;88:2460-2470.

47. Netea RT, Smits P, Lenders JW, et al. Does it matter whether blood pressure measurements are taken with subjects sitting or supine? $J$ Hypertens. 1998;16:263-268.

48. Netea RT, Elving LD, Lutterman JA, et al. Body position and blood pressure measurement in patients with diabetes mellitus. J Intern Med. 2002;251:393-399.

49. Netea RT, Lenders JW, Smits P, et al. Both body and arm position significantly influence blood pressure measurement. J Hum Hypertens. $2003 ; 17: 459-462$

50. Mitchell PL, Parlin RW, Blackburn H. Effect of vertical displacement of the arm on Indirect blood-pressure measurement. $N$ Engl J Med. 1964;271:72-74.

51. Webster J, Newnham D, Petrie JC, et al. Influence of arm position on measurement of blood pressure. Br Med J (Clin Res Ed). 1984;288:15741575 .

52. Parati G, Pomidossi G, Casadei R, et al. Lack of alerting reactions to intermittent cuff inflations during noninvasive blood pressure monitoring. Hypertension. 1985;7:597-601.

53. Veerman DP, Van Montfrans GA, Karemaker JM, et al. Inflating one's own cuff does not increase self-recorded blood pressure. J Hypertens. 1988;6(4):S77-S78.

54. Mejia AD, Egan BM, Schork NJ, et al. Artefacts in measurement of blood pressure and lack of target organ involvement in the assessment 
of patients with treatment-resistant hypertension. Ann Intern Med. 1990;112:270-277.

55. Wang JJ, Liu SH, Chern Ch I, et al. Development of an arterial applanation tonometer for detecting arterial blood pressure and volume. Biomed Eng Appl Basis Comm. 2004;16:322-330.

56. Langwieser N, Prechtl L, Meidert AS, Radial artery applanation tonometry for continuous noninvasive arterial blood pressure monitoring in the cardiac intensive care unit. Clin Res Cardiol. 2015;104:518-524.

57. Greiwe G, Tariparast PA, Behem C, et al. Is applanation tonometry a reliable method for monitoring blood pressure in morbidly obese patients undergoing bariatric surgery? British J. Anaesth. 2016;116:790-796.

58. Greiwe G, Hoffmann S, Herich L, et al. Comparison of blood pressure monitoring by applanation tonometry and invasively assessed blood pressure in cardiological patients. J Clin Monit Comput. 2017.

59. Cameron JD, Mc Grath BP, Dart AM, Use of radial artery applanation tonometry and a generalized transfer function to determine aortic pressure augmentation in subjects with treated hypertension. $J$ Am Coll Cardiol. 1998;32:1214-1220.

60. Nelson MR, Stepanek J, Cevette M, et al. Noninvasive measurement of central vascular pressures with arterial tonometry: clinical revival of the pulse pressure waveform? Mayo Clin Proc. 2010;85:460-472.

61. Langwieser N, Prechtl L, Meidert AS, et al. Radial artery applanation tonometry for continuous noninvasive arterial blood pressure monitoring in the cardiac intensive care unit. Clin Res Cardiol. 2015;104:518-524.

62. Greiwe G, Hoffmann S, Herich L, et al. Comparison of blood pressure monitoring by applanation tonometry and invasively assessed blood pressure in cardiological patients. J Clin Monit Comput. 2017.

63. Ilies C, Grudev G, Hedderich J, et al. Comparison of a continuous noninvasive arterial pressure device with invasive measurements in cardiovascular postsurgical intensive care patients: a prospective observational study. Eur J Anaesthesiol. 2015;32:20-28.

64. Stergiou GS, Kollias A, Destounis A, et al. Automated blood pressure measurement in atrial fibrillation: a systematic review and meta-analysis. J Hypertens. 2012;30:2074-2082.

65. Harju J, Vehkaoja A, Kumpulainen P, et al. Comparison of non-invasive blood pressure monitoring using modified arterial applanation tonometry with intra-arterial measurement. J Clin Monit Comput. 2018;32:13-22.

66. Imholz BP, Wieling W, Van Montfrans GA, et al. Fifteen years experience with finger arterial pressure monitoring: assessment of the technology. Cardiovasc Res. 1998;38:605-616.
67. Wesseling KH, De Wit B, Van Der Hoeven GMA, et al. Physiocal, calibrating finger vascular physiology for Finapres. Homeostasis. $1995 ; 36: 76-82$

68. Chung E, Chen G, Alexander B, et al. Non-invasive continuous blood pressure monitoring: A review of current applications. Front Med. 2013;7:91-101.

69. Milnor WR. Hemodynamics. Baltimore: Williams\&Wilkins; 1989.

70. Gratz I, Deal E, Spitz F, et al. Continuous non-invasive finger cuff CareTaker ${ }^{\circledR}$ comparable to invasive intra-arterial pressure in patients undergoing major intra-abdominal surgery. BMC Anesthesiol. 2017;17:48.

71. Imholz BP, Wieling W, Van Montfrans GA, et al. Fifteen years experience with finger arterial pressure monitoring: assessment of the technology. Cardiovasc Res. 1998;38:605-616.

72. Kim SH, Lilot M, Sidhu KS, et al. Accuracy and precision of continuous noninvasive arterial pressure monitoring compared with invasive arterial pressure: a systematic review and meta-analysis. Anesthesiology. 2014;120:1080-1097.

73. O Brien E, Waeber B, Parati G. Blood pressure measuring devices: recommendations of the European Society of Hypertension. BMJ. 2001;322:531-536.

74. Meng X, Zang G, Fan L, et al. Non-invasive monitoring of blood pressure using the Philips Intellivue MP50 monitor cannot replace invasive blood pressure techniques in surgery patients under general anesthesia. Experim Therapeut Med. 2013;6:9-14.

75. Thin, flexible, adhesive, continuous, cuffless blood pressure sensor.

76. Rheuban KS, Krupinski EA. Understanding Telehealth. McGraw-Hill Educ. 2018.

77. Norris AC. Essentials of Telemedicine and Telecare. John Wiley \& Sons, Ltd. 2002.

78. Weinstein RS, Lopez AM, Joseph BA. Applications that work: opportunities and barriers. Amer J Med. 2014;127:183-187.

79. Topouchian J, Agnoletti D, Blacher J, et al. Validation of four automatic devices for self-measurement of blood pressure according to the international protocol of the European Society of Hypertension. Vasc Health Risk Manag. 2011;7:709-717. 